\title{
Application of MIMO DF Equalization to High-Speed Off-Chip Communication
}

\author{
Lennert Jacobs *, Mamoun Guenach ${ }^{\dagger}$ and Marc Moeneclaey * \\ * Department of Telecommunications and Information Processing \\ Ghent University, Ghent, Belgium \\ Email: \{lennert.jacobs,marc.moeneclaey\}@telin.ugent.be \\ $\dagger$ Alcatel-Lucent Bell Labs, Antwerp, Belgium \\ Email: guenach@ieee.org
}

\begin{abstract}
In this contribution, we present a multiple-input multiple-output (MIMO) equalizer with decision feedback (DF) for high-speed chip-to-chip communication. We derive an elegant closed-form expression for the minimum mean square error (MMSE) equalization filters and show that the application of MIMO DF equalization (DFE) allows to significantly improve the reliability of high-speed communication over low-cost electrical interconnects.
\end{abstract}

\section{INTRODUCTION}

As the processing speed of integrated circuits (ICs) is ever increasing, the bit rates to be achieved by chip-to-chip communication need to scale accordingly. However, due to growing signal bandwidths, current electrical chip-to-chip interconnects start to suffer from high-frequency attenuation, caused by skin effect and dielectric loss. In addition to the intersymbol interference (ISI) resulting from channel dispersion, crosstalk (XT) originating from mutual coupling between neighboring wires further degrades the error performance. Hence, sophisticated equalization techniques are required to achieve multiGbit/s communication over low-cost electrical chip-to-chip interconnects. Most state-of-the-art transceivers apply nonlinear decision feedback equalization (DFE) or TomlinsonHirashima precoding (THP) to combat ISI [1]-[4]. In order to mitigate XT, a myriad of XT cancellation techniques have been proposed such as analog front-end design with infinite impulse response (IIR) networks [5], proper printed circuit board (PCB) design [6], capacitive crosstalk compensation [7], and wavelet based filtering [8]. In [9], it was shown that linear multiple-input multiple-output (MIMO) equalization is able to significantly outperform conventional single-input singleoutput (SISO) equalization by exploiting the XT signals rather than suppressing them.

In this work, we extend the results for linear MIMO equalization to MIMO DFE. Although the concept of MIMO DFE has been introduced in, e.g., [10], [11], we derive elegant closed-form matrix expressions for the feedforward and feedback equalizers, and show how the proposed equalization scheme allows to improve the reliability of high-speed offchip communication over low-cost electrical interconnects by simultaneously mitigating ISI and XT.

978-1-4799-8569-2/15/\$31.00 @2015 IEEE

\section{SYSTEM MODEL}

Fig. 1 exhibits a baseband communication scheme connecting $L$ adjacent transmitters to a MIMO DFE receiver through a typical electrical chip-to-chip interconnect consisting of $L$ parallel lanes. Each of the $L$ transmitters feeds a realvalued data symbol stream $\left\{a^{(l)}(k)\right\}$, with $1 \leq l \leq L$, to a pulse shaping filter $H_{\mathrm{tr}}(f)$ at a symbol rate $\overline{1} / T$. Assuming spatially and temporally independent data streams, i.e., $E\left[a^{\left(l_{1}\right)}\left(k_{1}\right) a^{\left(l_{2}\right)}\left(k_{2}\right)\right]=\sigma_{a}^{2} \delta_{l_{1}-l_{2}} \delta_{k_{1}-k_{2}}$, the average energy transmitted per symbol is given by

$$
E_{\mathrm{s}}=\sigma_{a}^{2} \int_{-\infty}^{+\infty}\left|H_{\mathrm{tr}}(f)\right|^{2} \mathrm{~d} f .
$$

The direct and XT channels between the transmitters and the receiver establish a MIMO link captured by the matrix $\mathbf{H}_{\mathrm{ch}}(f)$, the $(r, p)$-th entry of which is the frequency response $H_{\mathrm{ch}}^{(r, p)}(f)$ describing the channel between the $p$-th transmitter and the $r$-th receiver, with $1 \leq r, p \leq L$. The $L$ received signals, affected by channel dispersion and XT, are each filtered by an analog receiver filter $H_{\text {rec }}(f)$ and sampled at (a multiple of) the symbol rate $1 / T_{\mathrm{FF}}=N_{\mathrm{FF}} / T$; obviously, the sampling instants $\left\{m T_{\mathrm{FF}}+\varepsilon T\right\}$ depend on the sampling phase $\varepsilon$. The samples $n^{(1)}(m), \ldots, n^{(L)}(m)$ represent additive stationary noise at the receiver. The spatial diversity offered by the channel matrix $\mathbf{H}_{\mathrm{ch}}(f)$ can be exploited by introducing the MIMO equalization matrix $\mathbf{H}_{\mathrm{FF}}$ representing $L \times L$ fractionallyspaced feedforward equalizer filters, operating at the sampling rate $N_{\mathrm{FF}} / T$. The variables $u^{(l)}(k)$ based on which the symbol decisions are taken, are obtained by downsampling the outputs of $\mathbf{H}_{\mathrm{FF}}$ and by subtracting from the result the outputs of the MIMO equalization matrix $\mathbf{H}_{\mathrm{FB}}$ representing the $L \times L$ symbol-spaced feedback equalizer filters. Clearly, the feedforward equalizers operate at the sampling rate $N_{\mathrm{FF}} / T$, whereas the feedback equalizers operate at the symbol rate $1 / T$. Moreover, the feedback equalization filters need to be strictly causal, since only past symbol decisions can be applied to the inputs of $\mathbf{H}_{\mathrm{FB}}$.

If the off-diagonal equalizer filters are zero, the MIMO system from Fig. 1 degenerates to a conventional SISO DFE scheme. When all feedback filters have zero coefficients, the MIMO DFE scheme reduces to the linear MIMO equalization scheme from [9]. It should be noted that the analog front end of the proposed MIMO equalization scheme is identical to the one in the case of traditional SISO equalization. Due to the larger number of discrete-time feedforward and feedback 


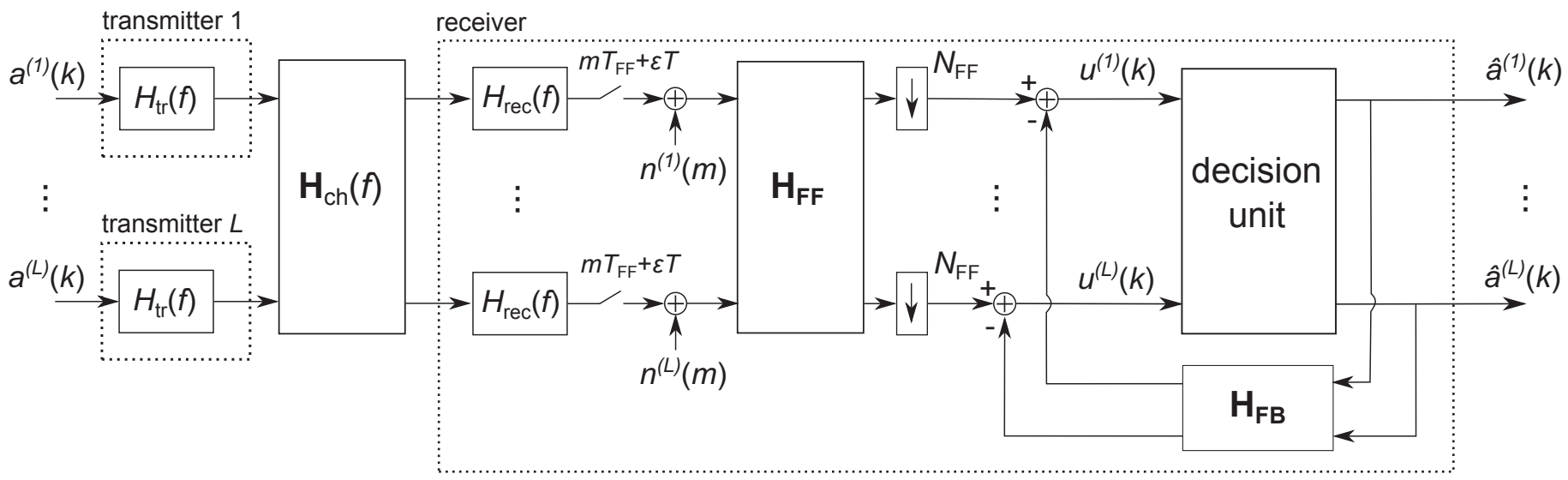

Figure 1. MIMO DFE scheme.

filters, however, the digital signal processing can suffer from increased computational complexity. Nonetheless, by taking the total number of filter coefficients as complexity measure, it is shown in Sec. IV that, for a given complexity, the proposed MIMO DFE scheme outperforms both SISO DFE equalization and linear MIMO equalization.

\section{MMSE MIMO DFE}

Assuming that the past symbol decisions which are applied to the feedback filter are correct, the outputs $\left\{u^{(l)}(k)\right\}$ of the MIMO DFE equalization scheme, with $1 \leq l \leq L$, are given by

$$
\begin{aligned}
u^{(l)}(k)=\sum_{p=1}^{L} \sum_{m} h^{(l, p)}(m) a^{(p)}(k-m) & \\
& -\sum_{p=1}^{L} \sum_{m>0} h_{\mathrm{FB}}^{(l, p)}(m) a^{(p)}(k-m) \\
& +\sum_{p=1}^{L} \sum_{m} h_{\mathrm{FF}}^{(l, p)}(m) n^{(p)}\left(k N_{\mathrm{FF}}-m\right),
\end{aligned}
$$

where $h_{\mathrm{FF}}^{(l, p)}(m)$ and $h_{\mathrm{FB}}^{(l, p)}(m)$ are the impulse responses of the equalizer filters between the $p$-th input and the $l$-th output of the equalization matrices $\mathbf{H}_{\mathrm{FF}}$ and $\mathbf{H}_{\mathrm{FB}}$, respectively, and the impulse response $h^{(l, p)}(m)$ is defined as

$$
h^{(l, p)}(m)=\sum_{q=1}^{L} \sum_{m_{1}} h_{\mathrm{FF}}^{(l, q)}\left(m_{1}\right) g^{(q, p)}\left(m N_{\mathrm{FF}}-m_{1}\right),
$$

where the sequences $\left\{g^{(q, p)}(m)\right\}$, with $1 \leq q, p \leq$ $L$, are obtained by sampling at instants $\left\{m \bar{T}_{\mathrm{FF}}+\varepsilon T\right\}$ the impulse responses of the corresponding cascades $H_{\mathrm{tr}}(f) H_{\mathrm{ch}}^{(q, p)}(f) H_{\mathrm{rec}}(f)$. Ideally, in the absence of noise, ISI and XT, we should have $u^{(l)}(k)=a^{(l)}(k)$.

In practice, the feedforward and feedback equalization filters are finite impulse response (FIR) filters with a limited number of filter taps; we assume $h_{\mathrm{FF}}^{(r, q)}(m)=0$ for $m \notin$ $\left[-L_{\mathrm{FF}, \min }, L_{\mathrm{FF}, \max }\right]$, yielding $L_{\mathrm{FF}}=L_{\mathrm{FF}, \min }+L_{\mathrm{FF}, \max }+1$ feedforward filter taps, whereas $h_{\mathrm{FB}}^{(r, q)}(m)=0$ for $m \notin$
[1, $\left.L_{\mathrm{FB}}\right]$, yielding $L_{\mathrm{FB}}$ feedback filter taps. In order to enable convenient matrix notation, we introduce the $L \times\left(L L_{\mathrm{FF}}\right)$ block matrix $\overline{\mathbf{H}}_{\mathrm{FF}}$ comprising all feedforward equalizer coefficients

$$
\overline{\mathbf{H}}_{\mathrm{FF}}=\left[\breve{\mathbf{H}}_{\mathrm{FF}}\left(-L_{\mathrm{FF}, \min }\right), \ldots, \breve{\mathbf{H}}_{\mathrm{FF}}\left(L_{\mathrm{FF}, \max }\right)\right],
$$

where the $(l, q)$-th entry of the $L \times L$ matrix $\breve{\mathbf{H}}_{\mathrm{FF}}(m)$ is given by $h_{\mathrm{FF}}^{(l, q)}(m)$. Similarly, the $(l, q)$-th entry of the $L \times L$ matrix $\breve{\mathbf{H}}_{\mathrm{FB}}(m)$ is given by $h_{\mathrm{FB}}^{(l, q)}(m)$, with $m=1, \ldots, L_{\mathrm{FB}}$. The samples $\left\{g^{(q, p)}(m)\right\}$ are included in the $\left(L L_{\mathrm{FF}}\right) \times L$ block matrix $\overline{\mathbf{G}}(m)$ as follows

$$
\overline{\mathbf{G}}(m)=\left[\begin{array}{c}
\breve{\mathbf{G}}\left(m N_{\mathrm{FF}}+L_{\mathrm{FF}, \min }\right) \\
\vdots \\
\breve{\mathbf{G}}\left(m N_{\mathrm{FF}}-L_{\mathrm{FF}, \max }\right)
\end{array}\right],
$$

where the $(q, p)$-th entry of the $L \times L$ matrix $\breve{\mathbf{G}}(m)$ is given by $g^{(q, p)}(m)$. The sequences $\left\{g^{(q, p)}(m)\right\}$ are assumed to have limited time duration, i.e., $g^{(q, p)}(m)=0$ for $m \notin$ $\left[-L_{g, \min }, L_{g, \max }\right]$ and for all $q$ and $p$, such that the number of non-zero matrices $\overline{\mathbf{G}}(m)$ is limited to the interval $\left[-L_{\mathrm{G}, \min }, L_{\mathrm{G}, \max }\right]$, where

$$
\left\{\begin{aligned}
L_{\mathrm{G}, \min } & =\left\lfloor\frac{L_{g, \min }+L_{\mathrm{FF}, \min }}{N_{\mathrm{FF}}}\right\rfloor \\
L_{\mathrm{G}, \max } & =\left\lfloor\frac{L_{g, \max }+L_{\mathrm{FF}, \max }}{N_{\mathrm{FF}}}\right\rfloor
\end{aligned}\right.
$$

Defining the $\left(L L_{\mathrm{FF}}\right)$-dimensional column vector $\overline{\mathbf{n}}(m)$ as

$$
\overline{\mathbf{n}}(m)=\left[\begin{array}{c}
\breve{\mathbf{n}}\left(m N_{\mathrm{FF}}+L_{\mathrm{FF}, \min }\right) \\
\vdots \\
\breve{\mathbf{n}}\left(m N_{\mathrm{FF}}-L_{\mathrm{FF}, \max }\right)
\end{array}\right],
$$

with the $p$-th element of $\breve{\mathbf{n}}(m)$ being given by $n^{(p)}(m)$, and introducing the $L$-dimensional column vectors $\mathbf{a}(k)$ and $\mathbf{u}(k)$, the $l$-th elements of which are given by $a^{(l)}(k)$ and $u^{(l)}(k)$, respectively, it follows that equations (2) and (3) can be written as

$$
\begin{aligned}
\mathbf{u}(k)= & \sum_{m \in \Psi_{\mathrm{G}}} \mathbf{H}(m) \mathbf{a}(k-m) \\
& -\sum_{m \in \Psi_{\mathrm{FB}}} \breve{\mathbf{H}}_{\mathrm{FB}}(m) \mathbf{a}(k-m)+\overline{\mathbf{H}}_{\mathrm{FF}} \overline{\mathbf{n}}(k),
\end{aligned}
$$


where $\Psi_{\mathrm{G}}=\left[-L_{\mathrm{G}, \min }, L_{\mathrm{G}, \max }\right], \Psi_{\mathrm{FB}}=\left[1, L_{\mathrm{FB}}\right]$, and

$$
\mathbf{H}(\mathrm{m})=\overline{\mathbf{H}}_{\mathrm{FF}} \overline{\mathbf{G}}(m) .
$$

It follows from (8) that the term in $\mathbf{u}(k)$ resulting from the additive noise $\overline{\mathbf{n}}(k)$ is affected by the MIMO feedforward equalization matrix $\overline{\mathbf{H}}_{\mathrm{FF}}$. Hence, contrary to MIMO DFE, MIMO feedforward equalization is expected to give rise to noise enhancement. Taking (8) and (9) into account, the error vector $\mathbf{e}(k)=\mathbf{u}(k)-\mathbf{a}(k)$ between the actual output $\mathbf{u}(k)$ and the target output $\mathbf{a}(k)$ can be written as

$$
\begin{aligned}
& \mathbf{e}(k)=\sum_{m \in \Psi_{\mathrm{FB}}}\left(\mathbf{H}(m)-\breve{\mathbf{H}}_{\mathrm{FB}}(m)\right) \mathbf{a}(k-m) \\
& \quad+\sum_{m \in \Psi_{\mathrm{FF}}}\left(\mathbf{H}(m)-\delta_{m} \mathbf{I}_{L}\right) \mathbf{a}(k-m)+\overline{\mathbf{H}}_{\mathrm{FF}} \overline{\mathbf{n}}(k),
\end{aligned}
$$

where $\Psi_{\mathrm{FF}}=\Psi_{\mathrm{G}} \backslash \Psi_{\mathrm{FB}}$. As a performance measure for the proposed equalization scheme, we introduce the normalized mean square error (MSE) caused by noise, ISI, and XT:

$$
\mathrm{MSE} \triangleq \frac{E\left[\|\mathbf{e}(k)\|^{2}\right]}{E\left[\|\mathbf{a}(k)\|^{2}\right]} .
$$

From (10), it follows that the MSE (11) reduces to

$$
\begin{aligned}
\mathrm{MSE} & =\frac{1}{L \sigma_{a}^{2}}\left[\sigma_{a}^{2} \sum_{m \in \Psi_{\mathrm{FB}}}\left\|\overline{\mathbf{H}}_{\mathrm{FF}} \overline{\mathbf{G}}(m)-\breve{\mathbf{H}}_{\mathrm{FB}}(m)\right\|^{2}\right. \\
& +\sigma_{a}^{2} \sum_{m \in \Psi_{\mathrm{FF}}}\left\|\overline{\mathbf{H}}_{\mathrm{FF}} \overline{\mathbf{G}}(m)-\delta_{m} \mathbf{I}_{L}\right\|^{2} \\
& \left.+\operatorname{tr}\left(\overline{\mathbf{H}}_{\mathrm{FF}} \mathbf{R}_{\overline{\mathbf{n}}} \overline{\mathbf{H}}_{\mathrm{FF}}^{\mathrm{T}}\right)\right]
\end{aligned}
$$

where the superscript $\mathrm{T}$ denotes matrix transpose and the $\left(L L_{\mathrm{FF}}\right) \times\left(L L_{\mathrm{FF}}\right)$ autocorrelation matrix $\mathbf{R}_{\overline{\mathbf{n}}}$ is defined as

$$
\mathbf{R}_{\overline{\mathbf{n}}} \triangleq E\left[\overline{\mathbf{n}}(m) \overline{\mathbf{n}}(m)^{\mathrm{T}}\right] .
$$

It is readily verified that for any given feedforward filter matrix $\overline{\mathbf{H}}_{\mathrm{FF}}$, the MSE (12) is minimized by selecting the feedback filter for $m \in \Psi_{\mathrm{FB}}$ as

$$
\breve{\mathbf{H}}_{\mathrm{FB}}(m)=\overline{\mathbf{H}}_{\mathrm{FF}} \overline{\mathbf{G}}(m) .
$$

Taking (14) into account, and considering the resulting similarity between (12) and [9, Eq. (12)], the minimum mean square error (MMSE) feedforward equalization matrix $\overline{\mathbf{H}}_{\mathrm{FF}, \mathrm{MMSE}}$ minimizing the MSE between the actual output vector $\mathbf{u}(k)$ and the target output vector $\mathbf{a}(k)$ can be shown to be elegantly expressed as

$$
\overline{\mathbf{H}}_{\mathrm{FF}, \mathrm{MMSE}}=\overline{\mathbf{G}}(0)^{\mathrm{T}} \mathbf{A}^{-1},
$$

where

$$
\mathbf{A} \triangleq \sum_{m \in \Psi_{\mathrm{FF}}} \overline{\mathbf{G}}(m) \overline{\mathbf{G}}(m)^{\mathrm{T}}+\frac{1}{\sigma_{a}^{2}} \mathbf{R}_{\overline{\mathbf{n}}}
$$

Hence, (14) fully eliminates the ISI and XT terms corresponding to $\mathbf{a}(k-m)$, with $m \in \Psi_{\mathrm{FB}}$, whereas (15) reduces the remaining ISI and XT for $m \in \Psi_{\mathrm{FF}}$ at the cost of noise enhancement. By substituting (14) and (15) in (12), the minimum MSE is shown to reduce to

$$
\mathrm{MSE}_{\min }=\frac{1}{L} \operatorname{tr}\left(\mathbf{I}_{L}-\mathbf{G}(0)^{\mathrm{T}} \mathbf{A}^{-1} \mathbf{G}(0)\right) \text {. }
$$

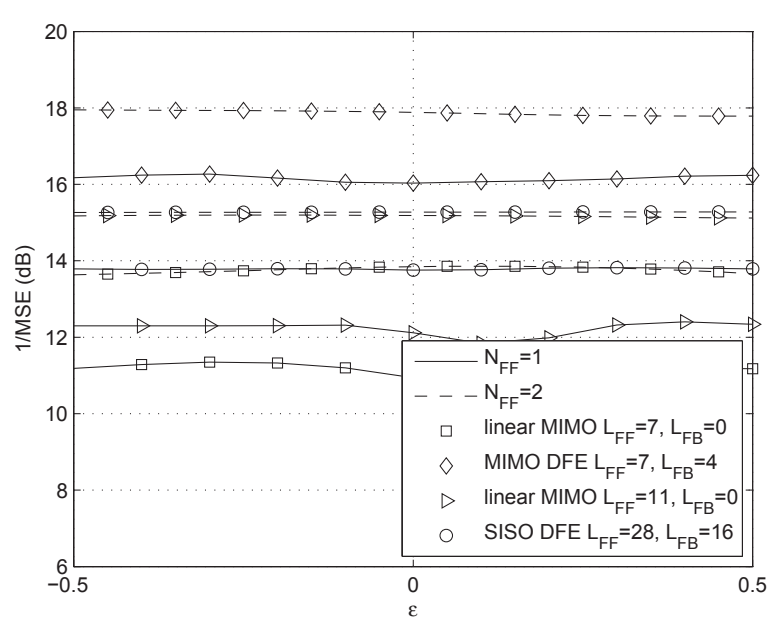

Figure 2. MSE for several symbol-spaced $\left(N_{\mathrm{FF}}=1\right)$ and fractionally-spaced $\left(N_{\mathrm{FF}}=2\right)$ equalization schemes.

\section{NUMERICAL RESULTS}

In this section, we illustrate the performance of the proposed equalization scheme on a $4 \times 4$ MIMO channel with strong XT, obtained from simulating an electrical chip-to-chip interconnect consisting of 4 adjacent stripline traces on a multilayer PCB. We assume unit-energy square-root raised-cosine transmit and receive filters with a $3 \mathrm{~dB}$ bandwidth $1 /(2 T)$ and a roll-off factor $\beta=0.3$. The considered constellation is 2-PAM. Furthermore, the noise samples $n^{(l)}(m)$ are spatially and temporally independent real-valued zero-mean Gaussian random variables with variance $N_{0} / 2$.

Under the assumption that $R_{\mathrm{b}}=50 \mathrm{Gbit} / \mathrm{s}$ and $E_{\mathrm{s}} / N_{0}=$ $20 \mathrm{~dB}$, we display in Fig. 2 the 1/MSE curves as a function of the sampling phase $\epsilon$ for a number of symbol-spaced $\left(N_{\mathrm{FF}}=\right.$ $1)$ and fractionally-spaced $\left(N_{\mathrm{FF}}=2\right)$ equalization schemes. Here, it is assumed that when $\varepsilon=0$, the impulse response corresponding to the frequency response $H_{\mathrm{tr}}(f) H_{\mathrm{ch}}^{(1,1)}(f) H_{\mathrm{rec}}(f)$ is sampled at the instant it reaches its maximum value. It can be observed from the figure that by adding $L_{\mathrm{FB}}=4$ feedback filter taps to a linear MIMO equalization scheme with $L_{\mathrm{FF}}=7$ (i.e., $L_{\mathrm{FF}, \min }=L_{\mathrm{FF}, \max }=3$ ) feedforward filter taps, the resulting MMSE MIMO DFE scheme outperforms the linear scheme by $5 \mathrm{~dB}$ when $N_{\mathrm{FF}}=1$ and by about $4 \mathrm{~dB}$ when $N_{\mathrm{FF}}=2$. Obviously, this performance improvement comes with the cost of increased computational complexity. A more fair comparison is obtained by considering equalization schemes with the same total number of filter taps. For instance, if we compare the MIMO DFE scheme with a linear equalization scheme with $L_{\mathrm{FF}}=11$ taps per feedforward filter, such that both schemes have a total number of $L_{\text {tot }}=11 \times 16=176$ taps, we notice that the performance improvement due to DFE reduces to about $4 \mathrm{~dB}$ and $2.6 \mathrm{~dB}$ when $N_{\mathrm{FF}}=1$ and $N_{\mathrm{FF}}=2$, respectively. However, given a total number of filter taps, it is still much more effective to use a MIMO DFE scheme than a linear MIMO equalization scheme. Additionally, if we compare the MIMO DFE scheme with a SISO DFE scheme with $L_{\mathrm{FF}, \max }=14, L_{\mathrm{FF}, \min }=13$, and $L_{\mathrm{FB}}=16$, such that $L_{\mathrm{tot}}=176$, we observe that the MIMO DFE scheme outperforms the SISO DFE scheme by 


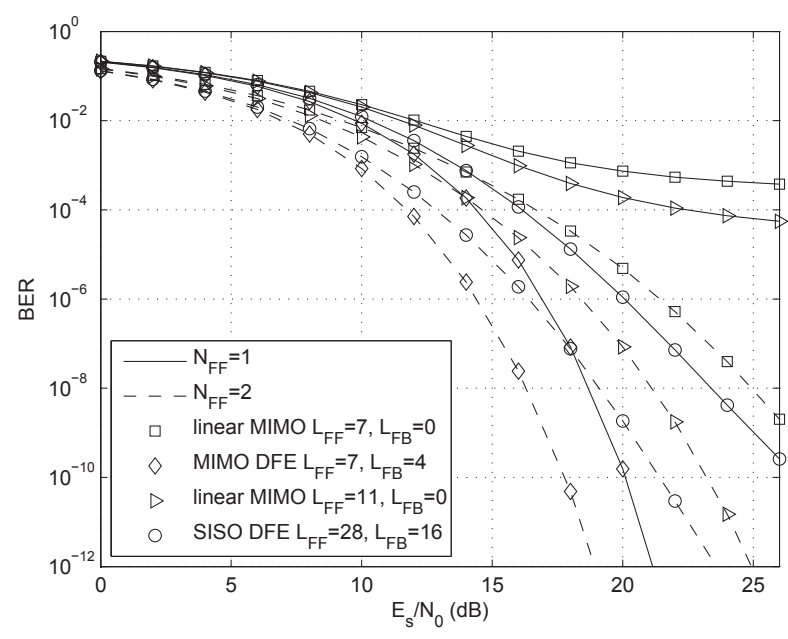

Figure 3. BER for several symbol-spaced $\left(N_{\mathrm{FF}}=1\right)$ and fractionally-spaced $\left(N_{\mathrm{FF}}=2\right)$ equalization schemes.

about $2.4 \mathrm{~dB}$ and $2.6 \mathrm{~dB}$ when $N_{\mathrm{FF}}=1$ and $N_{\mathrm{FF}}=2$, respectively. Note that the SISO DFE scheme treats the XT as additional Gaussian noise, as shown in [9]. Finally, by sampling at twice the sampling rate, the MSE performance of the MIMO DFE scheme is shown to be improved by about 2 $\mathrm{dB}$. The above observations indicate that (fractionally-spaced) MIMO DFE has the potential to significantly improve the performance of high-speed chip-to-chip communication over low-cost electrical interconnects.

In Fig. 3, we show the BER versus $E_{\mathrm{s}} / N_{0}$ for the equalization schemes from Fig. 2. For each scheme, we can obtain the optimal sampling phase from Fig. 2. However, since the MSE shows only minor variations as a function of $\varepsilon$, we select $\varepsilon=-0.25$ for all sampling schemes for the sake of simplicity. Considering a target BER of $10^{-12}$ and fractionally-spaced equalization $\left(N_{\mathrm{FF}}=2\right)$, the MIMO DFE scheme outperforms the linear MIMO equalization scheme (with $L_{\mathrm{FF}}=11$ ) by $6 \mathrm{~dB}$ and the SISO DFE scheme by about $4.5 \mathrm{~dB}$, despite the identical total number of filter taps. For symbol-spaced equalization $\left(N_{\mathrm{FF}}=1\right)$, the BER degradation between the MIMO and SISO DFE schemes is larger than for $N_{\mathrm{FF}}=2$, whereas the linear MIMO schemes do not achieve the target BER because of an error floor. Note that, due to error propagation, the actual BER will be slightly worse than the BER curves shown in Fig. 3. However, the conclusions drawn at the target BER of $10^{-12}$ remain valid, since for such low BERs, the effect of error propagation can be neglected.

\section{CONCLUSIONS}

In this contribution, we derived neat closed-form expressions for the MMSE equalization filters of a MIMO DFE scheme. We demonstrated the potential of the proposed scheme to improve the reliability of high-speed chip-to-chip communication over low-cost electrical interconnects. In particular, we showed that MIMO DFE allows to significantly outperform both linear MIMO equalization and conventional SISO DFE, even given a total number of filter taps.

\section{ACKNOWLEDGEMENTS}

Part of this research has been funded by the Interuniversity Attraction Poles Programme initiated by the Belgian Science Policy Office.

\section{REFERENCES}

[1] M. Kossel, T. Toifl, P. Francese, M. Brandli, C. Menolfi, P. Buchmann, L. Kull, T. Andersen, and T. Morf, "A $10 \mathrm{~Gb} / \mathrm{s}$ 8-Tap 6b 2-PAM/4PAM Tomlinson-Harashima Precoding Transmitter for Future MemoryLink Applications in 22-nm SOI CMOS," IEEE J. Solid-State Circuits, vol. 48, no. 12, pp. 3268-3284, Dec. 2013.

[2] H. Kimura, P. Aziz, T. Jing, A. Sinha, S. Kotagiri, R. Narayan, H. Gao, P. Jing, G. Hom, A. Liang, E. Zhang, A. Kadkol, R. Kothari, G. Chan, Y. Sun, B. Ge, J. Zeng, K. Ling, M. Wang, A. Malipatil, L. Li, C. Abel, and F. Zhong, "A $28 \mathrm{~Gb} / \mathrm{s} 560 \mathrm{~mW}$ Multi-Standard SerDes With SingleStage Analog Front-End and 14-Tap Decision Feedback Equalizer in 28 nm CMOS," IEEE J. Solid-State Circuits, vol. 49, no. 12, pp. 30913103, Dec 2014.

[3] J. Bulzacchelli, T. Beukema, D. Storaska, P. Hsieh, S. Rylov, D. Furrer, D. Gardellini, A. Prati, C. Menolfi, D. Hanson, J. Hertle, T. Morf, V. Sharma, R. Kelkar, H. Ainspan, W. Kelly, G. Ritter, J. Garlett, R. Callan, T. Toifl, and D. Friedman, "A 28Gb/s 4-tap FFE/15-tap DFE serial link transceiver in 32nm SOI CMOS technology," in IEEE Int. Solid-State Circuits Conf. Digest of Technical Papers (ISSCC), Feb 2012, pp. 324-326.

[4] M. Nazari and A. Emami-Neyestanak, "A 15-Gb/s 0.5-mW/Gbps TwoTap DFE Receiver With Far-End Crosstalk Cancellation," IEEE J. SolidState Circuits, vol. 47, no. 10, pp. 2420-2432, Oct. 2012.

[5] T. Oh and R. Harjani, "A 12-Gb/s Multichannel I/O Using MIMO Crosstalk Cancellation and Signal Reutilization in 65-nm CMOS," IEEE J. Solid-State Circuits, vol. 48, no. 6, pp. 1383-1397, Jun. 2013.

[6] P. Amleshi and C. Gao, "NEXT and FEXT characteristics and suppressions in dense 25Gbps+ backplane vias," in IEEE Int. Symp. on Electromagnetic Compatibility (EMC), Aug 2014, pp. 979-985.

[7] K.-D. Hwang and L.-S. Kim, "A 6.5-Gb/s 1-mW/Gb/s/CH Simple Capacitive Crosstalk Compensator in a 130-nm Process," IEEE Trans. Circuits Syst. II: Express Briefs, vol. 60, no. 6, pp. 302-306, June 2013.

[8] D. Shilpa and B. Uma, "A wavelet technique to minimize off-chip interconnect crosstalk," in Int. Conf. on Emerging Trends in Communication, Control, Signal Processing Computing Applications (C2SPCA), Oct 2013, pp. 1-5.

[9] L. Jacobs, M. Guenach, and M. Moeneclaey, "Linear MIMO equalization for high-speed chip-to-chip communication," in Proc. IEEE Int. Conf. Commun. (ICC) - to be published, 8-12 Jun. 2015.

[10] N. Al-Dhahir and A. Sayed, "The finite-length multi-input multi-output MMSE-DFE," IEEE Trans. Signal Process., vol. 48, no. 10, pp. 29212936, Oct 2000.

[11] A. Chevreuil and L. Vandendorpe, "MIMO MMSE-DFE: a general framework," in Proceedings Ninth IEEE SP Workshop on Statistical Signal and Array Processing, Sep 1998, pp. 368-371. 